\title{
The Marshall-Olkin extended generalized Gompertz distribution
}

\author{
Lazhar Benkhelifa ${ }^{1}$ \\ Laboratory of Applied Mathematics, Mohamed Khider University, Biskra, Algeria \\ Department of Mathematics and Informatics, Larbi Ben M'Hidi University, \\ Oum El-Bouaghi, Algeria
}

\begin{abstract}
A new four-parameter model called the Marshall-Olkin extended generalized Gompertz distribution is introduced. Its hazard rate function can be constant, increasing, decreasing, upside-down bathtub or bathtub-shaped depending on its parameters. Some mathematical properties of this model such as expansion for the density function, moments, moment generating function, quantile function, mean deviations, mean residual life, order statistics and Rényi entropy are derived. The maximum likelihood technique is used to estimate the unknown model parameters and the observed information matrix is determined. The applicability of the proposed model is shown by means of a real data set.
\end{abstract}

Key words: Marshall-Olkin distribution, generalized Gompertz distribution, moments, maximum likelihood estimation, observed information matrix

\section{Introduction}

The Gompertz distribution which was proposed by Gompertz (1825) plays an important role in modeling survival times, human mortality and actuarial data. It is applied in several areas such as biology, gerontology, computer and marketing science, among others. Some applications of the Gompertz distribution can be found in Johnson et al. (1995). In recent years, some extensions of the Gompertz distribution have been proposed in the literature. El-Gohary et al. (2013) introduced the generalized Gompertz (GG) distribution using the Lehmann (1953) alternative type I with cumulative distribution function (cdf)

$$
G(x)=\left(1-e^{-\frac{\beta}{\lambda}\left(e^{\lambda x}-1\right)}\right)^{\alpha}, x, \alpha, \beta, \lambda>0 .
$$

Jafari et al. (2014) defined the beta Gompertz distribution, using the beta-G generators defined by Eugene et al. (2002), with cdf

$$
G(x)=\frac{1}{B(\theta, \gamma)} \int_{0}^{1-e^{-\frac{\beta}{\lambda}\left(e^{\lambda x}-1\right)}} t^{\theta-1}(1-t)^{\gamma-1} d t, \quad x, \beta, \lambda, \theta, \gamma>0
$$


Roozegar et al. (2015) introduced the McDonald Gompertz distribution, using the McDonald-G generators defined by Alexander et al. (2012) with cdf

$$
G(x)=\frac{1}{B(\theta / \delta, \gamma)} \int_{0}^{\left(1-e^{-\frac{\beta}{\lambda}\left(e^{\lambda x}-1\right)}\right)^{\delta}} t^{\theta / \delta-1}(1-t)^{\gamma-1} d t, x, \beta, \lambda, \theta, \delta, \gamma>0 .
$$

da Silva et al. (2015) introduced the Kumaraswamy Gompertz distribution with cdf

$$
G(x)=1-\left[1-\left(1-e^{-\frac{\beta}{\lambda}\left(e^{\lambda x}-1\right)}\right)^{\theta}\right]^{\gamma}, \quad x, \beta, \lambda, \theta, \gamma>0
$$

A new method for adding a parameter to a family of distributions is proposed by Marshall and Olkin (1997). The resulting distribution, known as Marshall-Olkin extended (denoted by the prefix "MOE" for short) distribution. The MOE-G distribution gives more flexibility for modeling various types of real data in practice. For any baseline cdf $G(x), x \in \mathbb{R}$, the cdf of the MOE-G distribution is given by

$$
F(x)=\frac{G(x)}{\theta+\bar{\theta} G(x)}, \theta>0,
$$

where $\bar{\theta}=1-\theta$ is a tilt parameter. The probability density function (pdf) of the MOE-G distribution is

$$
f(x)=\frac{\theta g(x)}{[\theta+\bar{\theta} G(x)]^{2}}, \theta>0,
$$

where $g(x)=d G(x) / d x$ is the pdf of $G(x)$. The MOE-G distribution becomes the baseline distribution when $\theta=1$.

Several new distributions have been proposed by using the Marshall-Olkin method. For example, the MOE normal distribution has been proposed by Garcia et al. (2010), the MOE Burr type XII distribution has been introduced by Al-Saiari et al. (2014) (see also Kumar (2016), Ristíc and Kundu (2015) defined the MOE generalized exponential distribution, Okasha et al. (2016) introduced the MOE generalized linear exponential distribution and Benkhelifa (2017) introduced the MOE generalized Lindley distribution. Cordeiro et al. (2014) studied the general properties of the MOE-G distribution.

In this paper, we propose a new four-parameter continuous model, so-called the MOEGG distribution. We also provide several mathematical properties of the proposed model and apply it to real data.

The remainder of this paper is organized as follows. In Section 2, we define the MOEGG distribution and present some of its special cases. Some mathematical properties of the new model are given in Section 3. In Section 4 we estimate the model parameters by the maximum likelihood method and calculate the elements of the observed information matrix. In Section 5, we illustrate the flexibility of the new distribution by using a real data set. Finally, we give some conclusions in Section 6. 


\section{The MOEGG distribution}

By inserting (1) in (2), we get the cdf of the MOEGG distribution that is

The pdf corresponding to (3) is

$$
F(x)=\frac{\left(1-e^{-\frac{\beta}{\lambda}\left(e^{\lambda x}-1\right)}\right)^{\alpha}}{\theta+\bar{\theta}\left(1-e^{-\frac{\beta}{\lambda}\left(e^{\lambda x}-1\right)}\right)^{\alpha}}
$$

$$
f(x)=\frac{\alpha \beta \theta e^{\lambda x} e^{-\frac{\beta}{\lambda}\left(e^{\lambda x}-1\right)}\left(1-e^{-\frac{\beta}{\lambda}\left(e^{\lambda x}-1\right)}\right)^{\alpha-1}}{\left[\theta+\bar{\theta}\left(1-e^{-\frac{\beta}{\lambda}\left(e^{\lambda x}-1\right)}\right)^{\alpha}\right]^{2}}
$$

Hereafter, when $X$ is a random variable following the MOEGG distribution, it will be denoted by $X \sim \operatorname{MOEGG}(\alpha, \beta, \lambda, \theta)$. Plots of the MOEGG density for selected parameter values are displayed in Figure 1. We observe that the density function can take various forms, depending on the parameter values. It is evident that the MOEGG distribution is much more flexible than the GG distribution.

The hazard rate function defined by $h(x)=f(x) /[1-F(x)]$ is an important quantity characterizing lifetime phenomena. The hazard rate function for the MOEGG random variable is given by

$$
h(x)=\frac{\alpha \beta \theta e^{\lambda x} e^{-\frac{\beta}{\lambda}\left(e^{\lambda x}-1\right)}\left(1-e^{-\frac{\beta}{\lambda}\left(e^{\lambda x}-1\right)}\right)^{\alpha-1}}{\left[1-\left(1-e^{-\frac{\beta}{\lambda}\left(e^{\lambda x}-1\right)}\right)^{\alpha}\right]\left[\theta+\bar{\theta}\left(1-e^{-\frac{\beta}{\lambda}\left(e^{\lambda x}-1\right)}\right)^{\alpha}\right]}
$$

Plots of the hazard rate function of the MOEGG distribution for different values are given in Figure 2. We observe that the hazard rate functions of the MOEGG distribution can be constant, increasing, decreasing, upside-down bathtub and bathtub-shaped depending basically on the values of the parameters.

The MOEGG distribution includes as special sub-models the following distributions:

- If $\theta=1$, then we obtain the GG distribution.

- If $\theta=1$ and $\lambda$ tends to zero, then we get the generalized exponential distribution.

- If $\theta=1$ and $\alpha=1$, then we get the Gompertz distribution.

- If $\theta=1, \alpha=1$, and $\lambda$ tends to zero, then we get the exponential distribution.

- If $\alpha=1$, then we get the MOE Gompertz distribution.

- If $\lambda$ tends to zero, then we get the MOE generalized exponential distribution.

- If $\alpha=1$ and $\lambda$ tends to zero, then we get the MOE exponential distribution. 

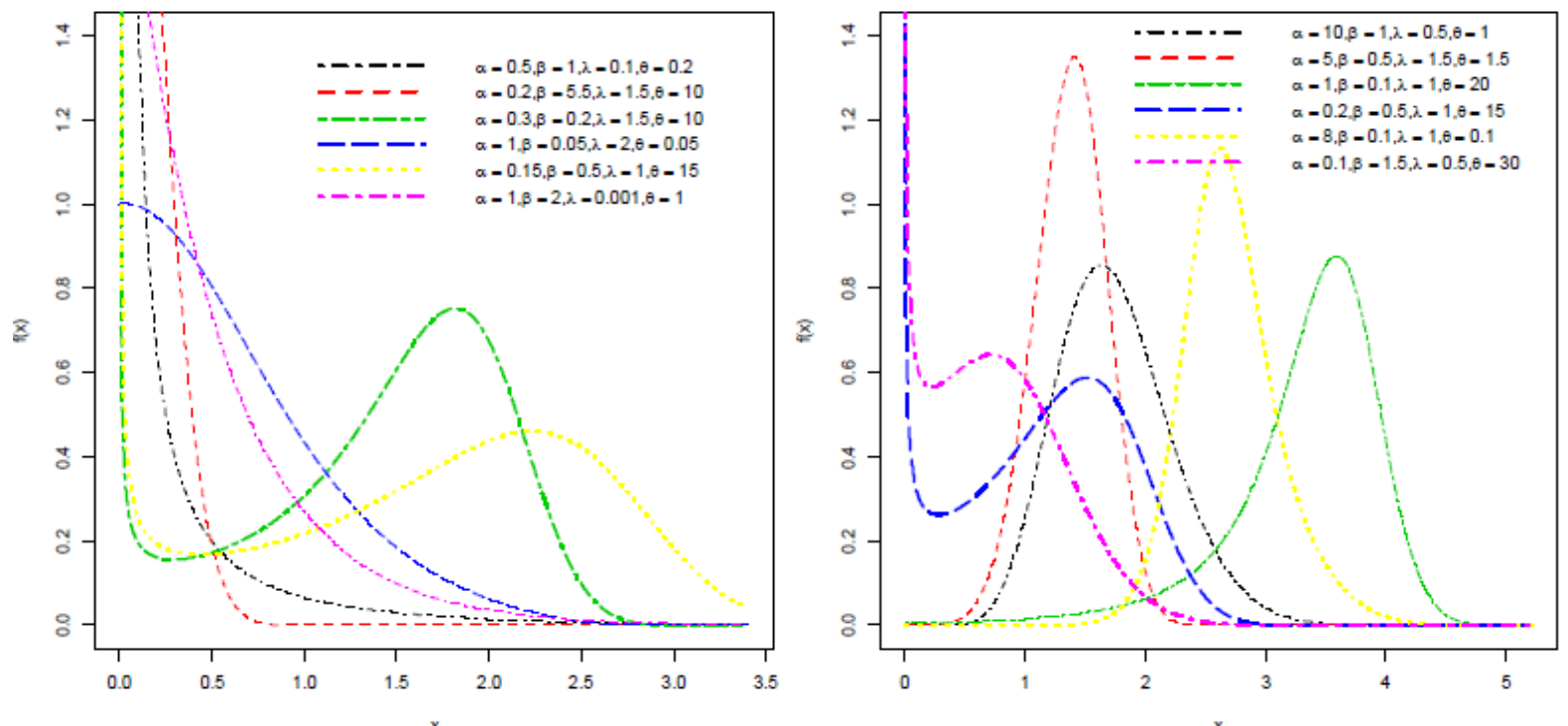

Figure 1: The MOEGG density function for some values of $\alpha, \beta, \lambda$ and $\theta$.
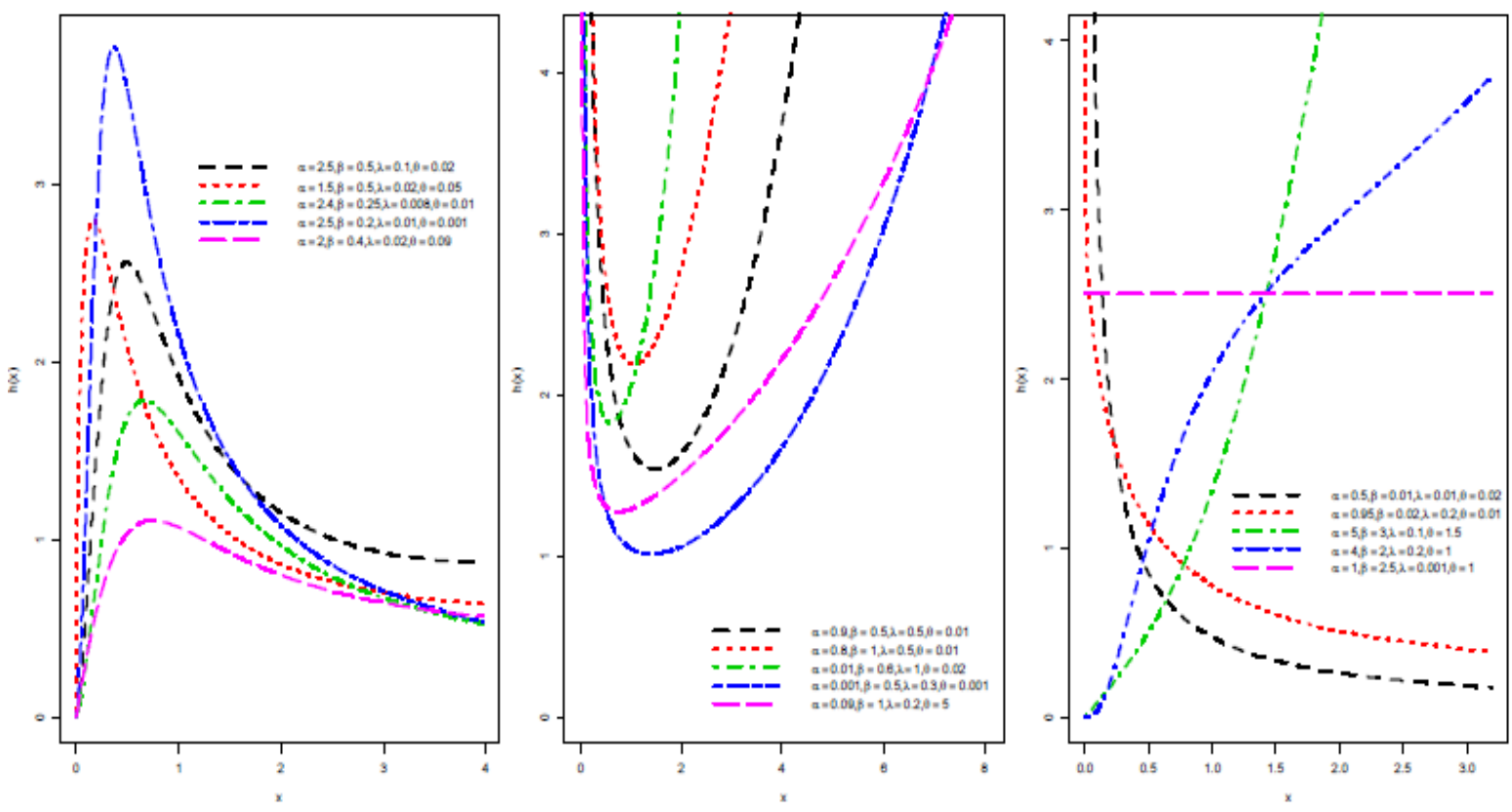

Figure 2: The MOEGG hazard rate function for some values of $\alpha, \beta, \lambda$ and $\theta$. 


\section{Mathematical properties}

In this section, we study some mathematical properties of the MOEGG model.

\subsection{Useful expansions}

In this subsection, we give useful expansions for the pdf and cdf of the MOEGG distribution. If $|z|<1$ and $\delta>0$ is a real non-integer, we have the expansion

$$
(1-z)^{-\delta}=\sum_{j=0}^{\infty}\left(\begin{array}{c}
\delta+j-1 \\
j
\end{array}\right) z^{j}
$$

We can rewrite the pdf of the MOEGG distribution (4) as

$$
f(x)=\frac{\alpha \beta \theta e^{\lambda x} e^{-\frac{\beta}{\lambda}\left(e^{\lambda x}-1\right)}\left(1-e^{-\frac{\beta}{\lambda}\left(e^{\lambda x}-1\right)}\right)^{\alpha-1}}{\left[1-\bar{\theta}\left(1-\left(1-e^{-\frac{\beta}{\lambda}\left(e^{\lambda x}-1\right)}\right)^{\alpha}\right)\right]^{2}} .
$$

Then, if $\theta \in(0,1)$, by applying the expansion (5), we get

$$
\begin{gathered}
{\left[1-\bar{\theta}\left(1-\left(1-e^{-\frac{\beta}{\lambda}\left(e^{\lambda x}-1\right)}\right)^{\alpha}\right)\right]^{-2}=\sum_{j=0}^{\infty}(\mathrm{j}+1) \bar{\theta}^{j}\left(1-\left(1-e^{-\frac{\beta}{\lambda}\left(e^{\lambda x}-1\right)}\right)^{\alpha}\right)^{j}} \\
=\sum_{j=0}^{\infty}(\mathrm{j}+1) \bar{\theta}^{j} \sum_{i=0}^{j}(-1)^{i}\left(\begin{array}{l}
j \\
i
\end{array}\right)\left(1-e^{-\frac{\beta}{\lambda}\left(e^{\lambda x}-1\right)}\right)^{\alpha i} .
\end{gathered}
$$

Therefore

$$
f(x)=\alpha \beta \theta e^{\lambda x} e^{-\frac{\beta}{\lambda}\left(e^{\lambda x}-1\right)} \sum_{j=0}^{\infty}(\mathrm{j}+1) \bar{\theta}^{j} \sum_{i=0}^{j}(-1)^{i}\left(\begin{array}{l}
j \\
i
\end{array}\right)\left(1-e^{-\frac{\beta}{\lambda}\left(e^{\lambda x}-1\right)}\right)^{\alpha(i+1)-1} .
$$

By interchanging $\sum_{j=0}^{\infty} \sum_{i=0}^{j}$ with $\sum_{i=0}^{\infty} \sum_{j=i}^{\infty}$ in the last equation, we get

$$
f(x)=\sum_{i=1}^{\infty} w_{i} g(x ; \alpha(i+1), \beta, \lambda),
$$

where

$$
w_{i}=\frac{\theta(-1)^{i}}{(i+1)} \sum_{j=1}^{\infty}\left(\begin{array}{l}
j \\
i
\end{array}\right)(j+1) \bar{\theta}^{j}, \text { for } i=0,1, \ldots
$$

By integrating (6), we obtain 


$$
F(x)=\sum_{i=1}^{\infty} w_{i} G(x ; \alpha(i+1), \beta, \lambda) .
$$

Otherwise, if $\theta>1$, we can rewrite the MOEGG density function (4) as

$$
f(x)=\frac{\alpha \beta \theta e^{\lambda x} e^{-\frac{\beta}{\lambda}\left(e^{\lambda x}-1\right)}\left(1-e^{-\frac{\beta}{\lambda}\left(e^{\lambda x}-1\right)}\right)^{\alpha-1}}{\theta\left[1-\left(1-\theta^{-1}\right)\left(1-e^{-\frac{\beta}{\lambda}\left(e^{\lambda x}-1\right)}\right)^{\alpha}\right]^{2}},
$$

then by using the expansion (5), we get

$$
f(x)=\sum_{i=1}^{\infty} v_{i} g(x ; \alpha(i+1), \beta, \lambda)
$$

where $v_{i}=\theta^{-1}\left(1-\theta^{-1}\right)^{i}$ for $i=0,1, \ldots$ By integrating (7), we obtain

$$
F(x)=\sum_{i=1}^{\infty} v_{i} G(x ; \alpha(i+1), \beta, \lambda) \text {. }
$$

It easy to verify that $\sum_{i=0}^{\infty} v_{i}=\sum_{i=0}^{\infty} w_{i}=1$. Equations (6) and (7) show that the MOEGG density function (4) is an infinite linear combination of GG density functions and have the same form except for the coefficients which are $w_{i}{ }^{\prime} \mathrm{s}$ in (6) and $v_{i}{ }^{\prime} \mathrm{s}$ in (7). Hence, several mathematical properties of the MOEGG distribution can be obtained directly from the properties of the GG distribution.

\subsection{Moments}

Let $X \sim \operatorname{MOEGG}(\alpha, \beta, \lambda, \theta)$. The rth moment of $X$, say $E\left(X^{r}\right)$, is given by

$$
E\left(X^{r}\right)=\int_{0}^{\infty} x^{r} f(x) d x
$$

From (7), if $\theta>1$, we have

$$
E\left(X^{r}\right)=\sum_{i=1}^{\infty} v_{i} E\left(X_{i+1}^{r}\right)
$$

where $X_{i+1}$ denotes a random variable having the GG density function $g(x ; \alpha(i+1), \beta, \lambda)$.

Then, we have

$$
E\left(X_{i+1}^{r}\right)=\alpha(i+1) \beta \int_{0}^{\infty} x^{r} e^{\lambda x} e^{-\frac{\beta}{\lambda}\left(e^{\lambda x}-1\right)}\left(1-e^{-\frac{\beta}{\lambda}\left(e^{\lambda x}-1\right)}\right)^{\alpha(i+1)-1} d x
$$


Since $0<e^{-\frac{\beta}{\lambda}\left(e^{\lambda x}-1\right)}<1$ for $x>0$, by applying the binomial expansion

$$
\begin{aligned}
& \left(1-e^{-\frac{\beta}{\lambda}\left(e^{\lambda x}-1\right)}\right)^{\alpha(i+1)-1} \text { given by } \\
& \left(1-e^{-\frac{\beta}{\lambda}\left(e^{\lambda x}-1\right)}\right)^{\alpha(i+1)-1}=\sum_{j=0}^{\infty}\left(\begin{array}{c}
\alpha(i+1)-1 \\
j
\end{array}\right)(-1)^{j} e^{-\frac{j \beta}{\lambda}\left(e^{\lambda x}-1\right)},
\end{aligned}
$$

we obtain

$$
E\left(X_{i+1}^{r}\right)=\alpha(i+1) \beta \sum_{j=0}^{\infty}\left(\begin{array}{c}
\alpha(i+1)-1 \\
j
\end{array}\right)(-1)^{j} e^{\frac{\beta}{\lambda}(j+1)} \int_{0}^{\infty} x^{r} e^{\lambda x} e^{-\frac{\beta}{\lambda}(j+1) e^{\lambda x}} d x .
$$

By substituting $z=e^{\lambda x}$ in the above integral, we get

$$
E\left(X_{i+1}^{r}\right)=\alpha(i+1) \beta \sum_{j=0}^{\infty}\left(\begin{array}{c}
\alpha(i+1)-1 \\
j
\end{array}\right) \frac{(-1)^{j} e^{\frac{\beta}{\lambda}(j+1)}}{\lambda^{r+1}} \int_{1}^{\infty}(\ln z)^{r} e^{-\frac{\beta}{\lambda}(j+1) z} d z
$$

By integration by parts, on the above integral, we get

$$
E\left(X_{i+1}^{r}\right)=\frac{\alpha(i+1) r !}{\lambda^{r}} \sum_{j=0}^{\infty}\left(\begin{array}{c}
\alpha(i+1)-1 \\
j
\end{array}\right) \frac{(-1)^{j} e^{\frac{\beta}{\lambda}(j+1)}}{(j+1)} E_{1}^{r-1}\left(\frac{\beta}{\lambda}(j+1)\right),
$$

where

$$
E_{S}^{k}(z)=\frac{1}{k !} \int_{1}^{\infty}(\ln x)^{k} x^{-s} e^{-z x} d x
$$

is the generalized integro-exponential function (see Milgram, 1985). Therefore

$$
E\left(X^{r}\right)=\sum_{i, j=0}^{\infty}\left(\begin{array}{c}
\alpha(i+1)-1 \\
j
\end{array}\right) \frac{(-1)^{j} r ! \alpha(i+1) v_{i} e^{\frac{\beta}{\lambda}(j+1)}}{(j+1) \lambda^{r}} E_{1}^{r-1}\left(\frac{\beta}{\lambda}(j+1)\right) .
$$

In a similar way, if $\theta \in(0,1)$, the $r$ th moment of $X$ is obtained from (6), by replacing $w_{i}$ with $v_{i}$ in the above equation.

The first four moments can be used to describe any data. The first moment or the mean is a measure of the center of the distribution. The second moment about the mean is equal to the variance which measures the spread of the distribution about the mean. The skewness measures the symmetry of a distribution while the kurtosis measures the peakedness of a distribution. The skewness and the kurtosis, respectively, are

$$
\gamma_{1}=\frac{\mu_{3}}{\mu_{2}^{3 / 2}} \text { and } \gamma_{2}=\frac{\mu_{4}}{\mu_{2}^{2}},
$$

where $\mu_{2}, \mu_{3}$ and $\mu_{4}$ are the second, third and fourth central moments.

Table 1 lists the first four moments, variance, skewness and kurtosis for selected values of the parameter $\theta$ of the MOEGG distribution for $\alpha=0.5, \beta=1$ and $\lambda=2$. 
Table 1: Moments of the MOEGG distribution.

\begin{tabular}{lcccc}
\hline$E\left(X^{r}\right)$ & $\theta=1.5$ & $\theta=5$ & $\theta=0.5$ & $\theta=0.1$ \\
\hline$E(X)$ & 0.37187 & 0.58696 & 0.20827 & 0.06695 \\
$E\left(X^{2}\right)$ & 0.22837 & 0.44545 & 0.10431 & 0.02598 \\
$E\left(X^{3}\right)$ & 0.16956 & 0.37802 & 0.06976 & 0.01583 \\
$E\left(X^{4}\right)$ & 0.14115 & 0.34539 & 0.05456 & 0.01183 \\
Variance & 0.09008 & 0.10093 & 0.06094 & 0.021498 \\
Skewness & 0.65217 & -0.06003 & 1.50583 & 121.95925 \\
Kurtosis & -0.40545 & -0.78352 & 89.26947 & 831.79977 \\
\hline
\end{tabular}

\subsection{Moment generating function}

Let $X \sim \operatorname{MOEGG}(\alpha, \beta, \lambda, \theta)$. The moment generating function $M(t)=E\left(e^{t X}\right)$ of $X$ is

$$
M(t)=\int_{0}^{\infty} e^{t x} f(x) d x .
$$

In this subsection, we give two representations for $M(t)$ only for the case $\theta>1$, since the case $\theta \in(0,1)$ is completely analogous.

A first representation, using the Maclaurin series expansion of an exponential function, is

$$
M(t)=\sum_{r=0}^{\infty} \frac{t^{r}}{r !} E\left(X^{r}\right)
$$

where

$$
E\left(X^{r}\right)=\sum_{i, j=0}^{\infty}\left(\begin{array}{c}
\alpha(i+1)-1 \\
j
\end{array}\right) \frac{(-1)^{j} r ! \alpha(i+1) v_{i} e^{\frac{\beta}{\lambda}(j+1)}}{(j+1) \lambda^{r}} E_{1}^{r-1}\left(\frac{\beta}{\lambda}(j+1)\right) .
$$

Then

$$
M(t)=\sum_{i, j, r=0}^{\infty}\left(\begin{array}{c}
\alpha(i+1)-1 \\
j
\end{array}\right) \frac{(-1)^{j} t^{r} \alpha(i+1) v_{i} e^{\frac{\beta}{\lambda}(j+1)}}{(j+1) \lambda^{r}} E_{1}^{r-1}\left(\frac{\beta}{\lambda}(j+1)\right) .
$$

A second representation for $M(t)$ follows from (7) as

where

$$
M(t)=\sum_{i=0}^{\infty} v_{i} M_{i+1}(t)
$$

$$
M_{i+1}(t)=\alpha(i+1) \beta \int_{0}^{\infty} e^{t x} e^{\lambda x} e^{-\frac{\beta}{\lambda}\left(e^{\lambda x}-1\right)}\left(1-e^{-\frac{\beta}{\lambda}\left(e^{\lambda x}-1\right)}\right)^{\alpha(i+1)-1} d x .
$$


By using the binomial series expansion, we get

$$
M_{i+1}(t)=\alpha(i+1) \beta \sum_{j=0}^{\infty}\left(\begin{array}{c}
\alpha(i+1)-1 \\
j
\end{array}\right)(-1)^{j} e^{\frac{\beta}{\lambda}(j+1)} \int_{0}^{\infty} e^{t x} e^{\lambda x} e^{-\frac{\beta}{\lambda}(j+1) e^{\lambda x}} d x .
$$

The change of variable $z=e^{\lambda x}$ yields

$$
M_{i+1}(t)=\frac{\alpha(i+1) \beta}{\lambda} \sum_{j=0}^{\infty}\left(\begin{array}{c}
\alpha(i+1)-1 \\
j
\end{array}\right)(-1)^{j} e^{\frac{\beta}{\lambda}(j+1)} \int_{1}^{\infty} z^{\frac{t}{\lambda}} e^{-\frac{\beta}{\lambda}(j+1) z} d z .
$$

Then, by setting $y=\frac{\beta}{\lambda}(j+1) z$, we obtain

$M_{i+1}(t)=\alpha(i+1)\left(\frac{\beta}{\lambda}\right)^{\frac{t}{\lambda}+1} \sum_{j=0}^{\infty}\left(\begin{array}{c}\alpha(i+1)-1 \\ j\end{array}\right) \frac{(-1)^{j} e^{\frac{\beta}{\lambda}(j+1)}}{(j+1)^{\frac{t}{\lambda}+1}} \Gamma\left(\frac{t}{\lambda}+1, \frac{\beta}{\lambda}(j+1)\right)$,

where $\Gamma(.,$.$) is the incomplete gamma function defined by$

$$
\Gamma(u, v)=\int_{v}^{\infty} x^{u-1} e^{-x} d x
$$

Therefore, if $\theta>1$, we have

$$
M(t)=\sum_{i, j=0}^{\infty}\left(\begin{array}{c}
\alpha(i+1)-1 \\
j
\end{array}\right)\left(\frac{\beta}{\lambda}\right)^{\frac{t}{\lambda}+1} \frac{(-1)^{j} \alpha(i+1) v_{i} e^{\frac{\beta}{\lambda}(j+1)}}{(j+1)^{\frac{t}{\lambda}+1}} \Gamma\left(\frac{t}{\lambda}+1, \frac{\beta}{\lambda}(j+1)\right) .
$$

\subsection{Quantile function}

The quantile function of the MOEGG distribution is given by

$$
Q(u)=F^{-1}(u)=\frac{1}{\lambda} \ln \left(1-\frac{\beta}{\lambda} \ln \left(1-\left(\frac{\theta u}{1-\bar{\theta} u}\right)^{\alpha}\right)\right), \quad u \in(0,1),
$$

where $F^{-1}($.$) is the inverse distribution function. It is easy to simulate the MOEGG$ distribution.

Let $U$ be a continuous uniform variable on the unit interval $(0,1)$. Thus, using the inverse transformation method, the random variable $X=Q(U) \sim \operatorname{MOEGG}(\alpha, \beta, \lambda, \theta)$. We can use this equation to generate random numbers from the MOEGG distribution when the parameters $\alpha, \beta$, $\lambda$ and $\theta$ are known. 


\subsection{Mean deviations}

Let $X \sim \operatorname{MOEGG}(\alpha, \beta, \lambda, \theta)$. The mean deviations of $X$ about the mean $\mu=E(X)$ and about the

median $M$ can be used as measures of spread in a population. They are defined by

$$
\delta_{1}=\int_{0}^{\infty}|x-\mu| f(x) d x \text { and } \delta_{2}=\int_{0}^{\infty}|x-M| f(x) d x
$$

respectively. These measures can be expressed as

$$
\delta_{1}=2 \mu F(\mu)-2 \mu+2 \int_{\mu}^{\infty} x f(x) d x \text { and } \delta_{2}=-\mu+2 \int_{M}^{\infty} x f(x) d x
$$

We consider only the case $\theta>1$, since we can replace $w_{i}$ by $v_{i}$ when $\theta \in(0,1)$. Then, from

(7), we have

$$
\int_{\eta}^{\infty} x f(x) d x=\alpha \beta \theta \sum_{i=0}^{\infty}(i+1) v_{i} \int_{\eta}^{\infty} x e^{\lambda x} e^{-\frac{\beta}{\lambda}\left(e^{\lambda x}-1\right)}\left(1-e^{-\frac{\beta}{\lambda}\left(e^{\lambda x}-1\right)}\right)^{\alpha(i+1)-1} d x
$$

By using the binomial series expansion of $\left(1-e^{-\frac{\beta}{\lambda}\left(e^{\lambda x}-1\right)}\right)^{\alpha(i+1)-1}$, we get

$$
\int_{\eta}^{\infty} x f(x) d x=\alpha \beta \theta \sum_{i, j=0}^{\infty}\left(\begin{array}{c}
\alpha(i+1)-1 \\
j
\end{array}\right)(-1)^{j}(i+1) v_{i} \int_{\eta}^{\infty} x e^{\lambda x} e^{-\frac{(j+1) \beta}{\lambda}\left(e^{\lambda x}-1\right)} d x
$$

By using the substitution $z=e^{\lambda x}$ in the above integral, we can get 


$$
\int_{\eta}^{\infty} x f(x) d x=\frac{\alpha \beta \theta}{\lambda^{2}} \sum_{i, j=0}^{\infty}\left(\begin{array}{c}
\alpha(i+1)-1 \\
j
\end{array}\right)(-1)^{j}(i+1) v_{i} e^{\frac{\beta}{\lambda}(j+1)} \int_{e^{\lambda \eta}}^{\infty} e^{-\frac{\beta}{\lambda}(j+1) z} \ln z d z
$$

By changing variable and integrating by parts yield

$$
\begin{aligned}
\int_{\eta}^{\infty} x f(x) d x= & \frac{\alpha \beta \theta}{\lambda^{2}} \sum_{i, j=0}^{\infty}\left(\begin{array}{c}
\alpha(i+1)-1 \\
j
\end{array}\right)(-1)^{j}(i+1) v_{i} e^{\frac{\beta}{\lambda}(j+1)}\left[\lambda \eta e^{-\frac{\beta}{\lambda}(j+1) e^{\lambda \eta}}\right. \\
& \left.+\Gamma\left(0, \frac{\beta}{\lambda}(j+1) e^{\lambda \eta}\right)\right]
\end{aligned}
$$

where $\Gamma(.,$.$) is the incomplete gamma function.$

Therefore

$$
\begin{aligned}
\delta_{1}=2 \mu F(\mu)- & 2 \mu+\frac{2 \alpha \beta \theta}{\lambda^{2}} \sum_{i, j=0}^{\infty}\left(\begin{array}{c}
\alpha(i+1)-1 \\
j
\end{array}\right)(-1)^{j}(i+1) v_{i} e^{\frac{\beta}{\lambda}(j+1)} \\
& \times\left[\lambda \mu e^{-\frac{\beta}{\lambda}(j+1) e^{\lambda \mu}}+\Gamma\left(0, \frac{\beta}{\lambda}(j+1) e^{\lambda \mu}\right)\right]
\end{aligned}
$$

and

$$
\begin{gathered}
\delta_{2}=-\mu+\frac{2 \alpha \beta \theta}{\lambda^{2}} \sum_{i, j=0}^{\infty}\left(\begin{array}{c}
\alpha(i+1)-1 \\
j
\end{array}\right)(-1)^{j}(i+1) v_{i} e^{\frac{\beta}{\lambda}(j+1)}\left[\lambda M e^{-\frac{\beta}{\lambda}(j+1) e^{\lambda M}}\right. \\
\left.+\Gamma\left(0, \frac{\beta}{\lambda}(j+1) e^{\lambda M}\right)\right] .
\end{gathered}
$$


Table 2 gives $\delta_{1}$ and $\delta_{2}$ for selected parameter values of the $\operatorname{MOEGG}(\alpha, \beta, \lambda, \theta)$ distribution.

Table 2: Means deviations of the MOEGG distribution.

\begin{tabular}{cccccc}
\hline$\alpha$ & $\beta$ & $\lambda$ & $\theta$ & $\delta_{1}$ & $\delta_{2}$ \\
\hline 2 & 1.5 & 0.5 & 0.5 & 0.31524 & 0.01137 \\
2 & 1.5 & 0.5 & 0.8 & 0.34505 & 0.10172 \\
2 & 1.5 & 0.5 & 5 & 0.40255 & 1.19131 \\
2 & 1.5 & 0.5 & 15 & 0.39132 & 1.50232 \\
\hline
\end{tabular}

\subsection{Mean residual life}

The mean residual life, also known as the mean remaining life, plays an important role in many fields such as industrial reliability, biomedical science, life insurance and demography among others. The mean residual life function, at point $t \geq 0$, of a lifetime random variable $X \sim$ $\operatorname{MOEGG}(\alpha, \beta, \lambda, \theta)$ is

$$
\begin{aligned}
& \mu(t)=\frac{1}{1-F(t)} \int_{t}^{\infty}[1-F(x)] d x \\
& =\frac{\theta+\bar{\theta}\left(1-e^{-\frac{\beta}{\lambda}\left(e^{\lambda t}-1\right)}\right)^{\alpha}}{-1+2 \theta+\bar{\theta}\left(1-e^{-\frac{\beta}{\lambda}\left(e^{\lambda t}-1\right)}\right)^{\alpha}} \int_{t}^{\infty-1+2 \theta+\bar{\theta}\left(1-e^{-\frac{\beta}{\lambda}\left(e^{\lambda x}-1\right)}\right)^{\alpha}} d x . \\
& \theta+\bar{\theta}\left(1-e^{-\frac{\beta}{\lambda}\left(e^{\lambda x}-1\right)}\right)^{\alpha}
\end{aligned}
$$

Numerical values of $\mu(5)$ and $\mu(0.5)$ for some values of the parameters $\alpha, \beta, \lambda$ and $\theta$ are presented in Table 3.

Table 3: Mean residual life of the MOEGG distribution.

\begin{tabular}{llllll}
\hline$\alpha$ & $\beta$ & $\lambda$ & $\theta$ & $\mu(5)$ & $\mu(0.5)$ \\
\hline 2.5 & 1.5 & 0.5 & 0.1 & 0.05543 & 0.29961 \\
0.5 & 0.25 & 0.3 & 0.1 & 0.71577 & 1.09276 \\
2.5 & 1.5 & 0.5 & 0.8 & 0.05029 & 0.47139 \\
0.5 & 0.25 & 0.3 & 0.8 & 0.72227 & 1.57295 \\
2.5 & 1.5 & 0.5 & 2.5 & 0.04995 & 0.67338 \\
0.5 & 0.25 & 0.3 & 2.5 & 0.73767 & 2.21551 \\
2.5 & 1.5 & 0.5 & 8.5 & 0.04984 & 0.95539 \\
0.5 & 0.25 & 0.3 & 8.5 & 0.78810 & 3.16767 \\
\hline
\end{tabular}




\subsection{Rényi entropy}

The entropy of a random variable is a measure of uncertainty variation and has been used in various situations in science and engineering. In the literature, several measures of entropy have been studied. Here, we give the Rényi entropy for the MOEGG distribution. The Rényi entropy for the MOEGG distribution is

$$
I_{R}(s)=\frac{1}{1-s} \ln \left(\int_{0}^{\infty} f^{s}(x) d x\right), s>0, s \neq 1,
$$

where

$$
f^{s}(x)=\frac{(\alpha \beta \theta)^{s} e^{\lambda s x} e^{-\frac{s \beta}{\lambda}\left(e^{\lambda x}-1\right)}\left(1-e^{-\frac{\beta}{\lambda}\left(e^{\lambda x}-1\right)}\right)^{s(\alpha-1)}}{\left[\theta+\bar{\theta}\left(1-e^{-\frac{\beta}{\lambda}\left(e^{\lambda x}-1\right)}\right)^{\alpha}\right]^{2 s}} .
$$

By applying the expansion (5) to the denomination of the previous equation, we can rewrite after some algebra $f^{s}(x)$, for $\theta \in(0,1)$, as

$$
f^{s}(x)=(\alpha \beta \theta)^{s} \sum_{k=0}^{\infty} \sum_{i=0}^{\infty} \sum_{j=i}^{\infty}\left(\begin{array}{c}
2 s+j-1 \\
j
\end{array}\right)\left(\begin{array}{l}
j \\
i
\end{array}\right)\left(\begin{array}{c}
\alpha i \\
k
\end{array}\right) \bar{\theta}^{j}(-1)^{k+i} e^{\lambda s x} e^{-\frac{\beta}{\lambda}(k+s)\left(e^{\lambda x}-1\right)},
$$

and for $\theta>1$, we get

$$
\begin{gathered}
f^{s}(x)=\left(\frac{\alpha \beta}{\theta}\right)^{s} \sum_{k=0}^{\infty} \sum_{i=0}^{\infty} \sum_{j=i}^{\infty}\left(\begin{array}{c}
2 s+j-1 \\
j
\end{array}\right)\left(\begin{array}{c}
\alpha(j+s)-s \\
k
\end{array}\right) \\
\times\left(1-\theta^{-1}\right)^{j}(-1)^{k} e^{\lambda s x} e^{-\frac{\beta}{\lambda}(k+s)\left(e^{\lambda x}-1\right)} .
\end{gathered}
$$

Thus, the Rényi entropy is

where, if $\theta \in(0,1)$

$$
I_{R}(s)=\frac{1}{1-s} \ln \left(\int_{0}^{\infty} f^{s}(x) d x\right)
$$

$$
\int_{0}^{\infty} f^{s}(x) d x=(\alpha \beta \theta)^{s} \sum_{k=0}^{\infty} \sum_{i=0}^{\infty} \sum_{j=i}^{\infty}\left(\begin{array}{c}
2 s+j-1 \\
j
\end{array}\right)\left(\begin{array}{l}
j \\
i
\end{array}\right)\left(\begin{array}{c}
\alpha i \\
k
\end{array}\right) \frac{\bar{\theta}^{j}(-1)^{k+i}}{\lambda(k+s)^{s}} e^{\frac{\beta}{\lambda}(k+s)} \Gamma\left(s, \frac{\beta}{\lambda}(k+s)\right),
$$

and if $\theta>1$

$$
\begin{aligned}
\int_{0}^{\infty} f^{s}(x) d x= & (\lambda \alpha)^{s} \sum_{k=0}^{\infty} \sum_{i=0}^{\infty} \sum_{j=i}^{\infty}\left(\begin{array}{c}
2 s+j-1 \\
j
\end{array}\right)\left(\begin{array}{c}
\alpha(j+s)-s \\
k
\end{array}\right) \\
& \times \frac{\left(1-\theta^{-1}\right)^{j}(-1)^{k}}{\lambda(\theta k+\theta s)^{s}} e^{\frac{\beta}{\lambda}(k+s)} \Gamma\left(s, \frac{\beta}{\lambda}(k+s)\right),
\end{aligned}
$$

with $\Gamma(.,$.$) is the incomplete gamma function.$ 
Table 4 gives $I_{R}(0.2)$ of the MOEGG distribution for different choices of parameters $\alpha, \beta, \lambda$ and $\theta$.

Table 4: Rényi entropy of the MOEGG distribution.

\begin{tabular}{|l|l|l|l|l|}
\hline$\alpha$ & $\beta$ & $\lambda$ & $\theta$ & $I_{R}(0.2)$ \\
\hline 5 & 2 & 1.5 & 0.1 & 0.05879 \\
\hline 0.2 & 0.7 & 0.9 & 0.1 & 0.12745 \\
\hline 5 & 2 & 1.5 & 0.8 & 0.14771 \\
\hline 0.2 & 0.7 & 0.9 & 0.8 & 0.42179 \\
\hline 5 & 2 & 1.5 & 1.2 & 0.15798 \\
\hline 0.2 & 0.7 & 0.9 & 1.2 & 0.47002 \\
\hline 5 & 2 & 1.5 & 7.5 & 0.17568 \\
\hline 0.2 & 0.7 & 0.9 & 7.5 & 0.62701 \\
\hline
\end{tabular}

\subsection{Order statistics}

The order statistics play an important role in reliability and life testing. Let $\mathrm{X}_{1}, \ldots, \mathrm{X}_{\mathrm{n}}$ be a simple random sample from MOEGG distribution with cdf and pdf as in (3) and (4), respectively. Let $\mathrm{X}_{1, n}, \ldots, \mathrm{X}_{n, n}$ denote the order statistics obtained from this sample. In reliability literature, the $k$ th order statistic, say $\mathrm{X}_{k, n}$, denotes the lifetime of an (n-k+1)-out-of- $n$ system which consists of $n$ independent and identically components. The pdf of $\mathrm{X}_{k, n}$, is given by

$$
f_{k, n}(x)=\frac{n !}{(n-k) !(k-1) !} f(x)[F(x)]^{k-1}[1-F(x)]^{n-k}, \quad \text { for } k=1, \ldots, n \text {. }
$$

Since $0<F(x)<1$ for $x>0$, then by using the binomial series expansion of $[1-F(x)]^{n-k}$, we obtain

$$
f_{k, n}(x)=\frac{n !}{(n-k) !(k-1) !} \sum_{j=0}^{n-k}\left(\begin{array}{c}
n-k \\
j
\end{array}\right)(-1)^{j} f(x)[F(x)]^{k+j-1} .
$$

Then, from (2), we have

$$
f_{k, n}(x)=\frac{\theta n ! g(x)}{(n-k) !(k-1) !} \sum_{j=0}^{n-k}\left(\begin{array}{c}
n-k \\
j
\end{array}\right)(-1)^{j} \frac{[G(x)]^{k+j-1}}{[\theta+\bar{\theta} G(x)]^{k+j+1}} .
$$

For $\theta \in(0,1)$, by using expansion (5) in the above equation and after some algebra, we get

$$
f_{k, n}(x)=\sum_{i=0}^{\infty} \sum_{j=0}^{n-k} \sum_{l=0}^{i} c_{i j l} g(x ; \alpha(k+j+l), \beta, \lambda),
$$

where

$$
c_{i j l}=\frac{n ! \theta(1-\theta)^{i}\left(\begin{array}{c}
n-k \\
j
\end{array}\right)\left(\begin{array}{l}
i \\
l
\end{array}\right)\left(\begin{array}{c}
k+j+1 \\
i
\end{array}\right)(-1)^{j+l}}{(k-1) !(n-k) !(k+j+l)}, \quad \text { for } k=1, \ldots, n \text {. }
$$

In a similar way, if $\theta>1$, we get 


$$
f_{k, n}(x)=\sum_{i=0}^{\infty} \sum_{j=0}^{n-k} t_{i j} g(x ; \alpha(k+j+i), \beta, \lambda)
$$

where

$$
t_{i j}=\frac{n !(\theta-1)^{i}\left(\begin{array}{c}
n-k \\
j
\end{array}\right)\left(\begin{array}{c}
i+j+k \\
i
\end{array}\right)(-1)^{j}}{\theta^{i+j+k}(k-1) !(n-k) !(k+j+i)}, \quad \text { for } k=1, \ldots, n
$$

It is clear that

$$
\sum_{i=0}^{\infty} \sum_{j=0}^{n-k} \sum_{l=0}^{i} c_{i j l}=\sum_{i=0}^{\infty} \sum_{j=0}^{n-k} t_{i j}=1
$$

Equations (8) and (9) show that the pdf of the MOEGG order statistics is an infinite linear combination of GG density functions. So, several mathematical properties of the these order statistics can be obtained directly from the properties of the GG such as the ordinary moments, inverse and factorial moments, moment generating function, mean deviations. The $r$ th moment of the $k$ th order statistic is determined from (8) and (9) as

$$
E\left(X_{k, n}^{r}\right)=\sum_{i=0}^{\infty} \sum_{j=0}^{n-k} \sum_{l=0}^{i} c_{i j l} E\left(X_{k+j+l}^{r}\right), \quad \text { if } \theta \in(0,1),
$$

and

$$
E\left(X_{k, n}^{r}\right)=\sum_{i=0}^{\infty} \sum_{j=0}^{n-k} t_{i j} E\left(X_{k+j+i}^{r}\right), \quad \text { if } \theta>1,
$$

where

$$
E\left(X_{k+j+m}^{r}\right)=\frac{\alpha(k+j+m) r !}{\lambda^{r}} \sum_{j=0}^{\infty}\left(\begin{array}{c}
\alpha(k+j+m)-1 \\
j
\end{array}\right) \frac{(-1)^{j} e^{\frac{\beta}{\lambda}(j+1)}}{(j+1)} E_{1}^{r-1}\left(\frac{\beta}{\lambda}(j+1)\right) .
$$

\subsection{Stochastic orderings}

Stochastic order is the most common notion of comparison of random variables. This concept can be applied in several areas, such as insurance, operations research; queuing theory, survival analysis and reliability theory (see Shaked and Shanthikumar, 2007). Let $X$ and $Y$ are two random variables with distribution functions $F$ and $G$ and density functions $f$ and $g$, respectively. We say that 
- $X$ is smaller than $Y$ in the stochastic order, denoted by $X \leq_{s t} Y$, if $F(x) \leq$ $G(x)$ for all $x$.

- $X$ is smaller than $Y$ in the likelihood ratio order, denoted by $X \leq_{l r} Y$, if $f(x) / g(x)$ is decreasing in $x$.

- $X$ is smaller than $Y$ in the hazard rate order, denoted by $X \leq_{h r} Y$, if $(1-F(x)) /(1-G(x))$ is decreasing in $x$.

- $X$ is smaller than $Y$ in the reversed hazard rate order, denoted by

$X \leq_{r h r} Y$, if $F(x) / G(x)$ is decreasing in $x$.

We have the following implications (see Shaked and Shanthikumar, 2007)

$$
X \leq_{r h r} Y \Leftarrow X \leq_{l r} Y \Rightarrow X \leq_{h r} Y \Rightarrow X \leq_{s t} Y .
$$

Theorem. Let $X \sim \operatorname{MOEGG}\left(\alpha, \beta, \lambda, \theta_{1}\right)$ and let $Y \sim \operatorname{MOEGG}\left(\alpha, \beta, \lambda, \theta_{2}\right)$. If $\theta_{1}<\theta_{2}$, then

$$
X \leq_{r h r} Y \Leftarrow X \leq_{l r} Y \Rightarrow X \leq_{h r} Y \Rightarrow X \leq_{s t} Y .
$$

Proof. We have

$$
\frac{f(x)}{g(x)}=\frac{\theta_{1}}{\theta_{2}}\left[\frac{1-\bar{\theta}_{2}\left(1-(1-t)^{\alpha}\right)}{1-\bar{\theta}_{1}\left(1-(1-t)^{\alpha}\right)}\right]^{2},
$$

where $t=e^{-\frac{\beta}{\lambda}\left(e^{\lambda x}-1\right)}$. It easy to verify

$$
\frac{d}{d x}\left[\frac{f(x)}{g(x)}\right]=2 \frac{\theta_{1}}{\theta_{2}}\left(\theta_{1}-\theta_{2}\right) \frac{\left(\theta_{2}+\bar{\theta}_{2}(1-t)^{\alpha}\right) \alpha \beta e^{\lambda x} t(1-t)^{\alpha-1}}{\left(\theta_{1}+\bar{\theta}_{1}(1-t)^{\alpha}\right)^{3}} .
$$

Then, if $\theta_{1}<\theta_{2}$, we have $\frac{d}{d x}\left[\frac{f(x)}{g(x)}\right]<0$. So, $\frac{f(x)}{g(x)}$ is decreasing in $x$ i.e., $X \leq_{l r} Y$. From the implications (10), we get the remaining statements.

\section{Estimation}

In this section we present the maximum likelihood estimate (MLE) and derive the asymptotic confidence intervals of the unknown parameter vector $\boldsymbol{\Theta}=(\alpha, \beta, \lambda, \theta)^{T}$ of the MOEGG distribution. Let $x_{1}, \ldots, x_{n}$ be a random sample of size $n$ from MOEGG distribution with pdf (4), then the likelihood function is

$$
L(\boldsymbol{\Theta})=\prod_{i=1}^{n} \frac{\alpha \beta \theta e^{\lambda x_{i}} e^{-\frac{\beta}{\lambda}\left(e^{\lambda x_{i}-1}\right)}\left(1-e^{-\frac{\beta}{\lambda}\left(e^{\lambda x_{i}-1}\right)}\right)^{\alpha-1}}{\left[\theta+\bar{\theta}\left(1-e^{-\frac{\beta}{\lambda}\left(e^{\lambda x_{i}}-1\right)}\right)^{\alpha}\right]^{2}} .
$$

Taking the logarithm of equation (11), we get the log-likelihood function 


$$
\begin{aligned}
\ell(\Theta)=n \ln \alpha & +n \ln \beta+n \ln \theta+\lambda \sum_{i=1}^{n} x_{i}-\frac{\beta}{\lambda} \sum_{i=1}^{n}\left(e^{\lambda x_{i}}-1\right) \\
& +(\alpha-1) \sum_{i=1}^{n} \ln \left(1-e^{-\frac{\beta}{\lambda}\left(e^{\lambda x_{i}}-1\right)}\right) \\
& -2 \sum_{i=1}^{n} \ln \left(\theta+\bar{\theta}\left(1-e^{-\frac{\beta}{\lambda}\left(e^{\lambda x_{i}-1}\right)}\right)^{\alpha}\right)
\end{aligned}
$$

The components of the score vector are given, by taking the partial derivatives of $\ell(\boldsymbol{\Theta})$ with respect to $\alpha, \beta, \lambda$ and $\theta$, as follows

$$
\begin{aligned}
& U_{\alpha}=\frac{n}{\alpha}+\sum_{i=1}^{n} \ln \left(1-e^{-\frac{\beta}{\lambda}\left(e^{\left.\lambda x_{i}-1\right)}\right)}-2 \sum_{i=1}^{n} \frac{\bar{\theta}\left(1-e^{-\frac{\beta}{\lambda}\left(e^{\lambda x_{i}-1}\right)}\right)^{\alpha} \ln \left(1-e^{-\frac{\beta}{\lambda}\left(e^{\lambda x_{i}-1}\right)}\right)}{\theta+\bar{\theta}\left(1-e^{-\frac{\beta}{\lambda}\left(e^{\lambda x_{i}}-1\right)}\right)^{\alpha}},\right. \\
& U_{\beta}=\frac{n}{\beta}-\frac{1}{\lambda} \sum_{i=1}^{n}\left(e^{\lambda x_{i}}-1\right)+(\alpha-1) \sum_{i=1}^{n} \frac{\left(e^{\lambda x_{i}}-1\right) e^{-\frac{\beta}{\lambda}\left(e^{\lambda x_{i}-1}\right)}}{\lambda\left(1-e^{-\frac{\beta}{\lambda}\left(e^{\left.\lambda x_{i}-1\right)}\right)}\right.} \\
& -2 \sum_{i=1}^{n} \frac{\bar{\theta} \alpha\left(e^{\lambda x_{i}}-1\right) e^{-\frac{\beta}{\lambda}\left(e^{\lambda x_{i}-1}\right)}\left(1-e^{-\frac{\beta}{\lambda}\left(e^{\lambda x_{i}-1}\right)}\right)^{\alpha-1}}{\lambda\left(\theta+\bar{\theta}\left(1-e^{-\frac{\beta}{\lambda}\left(e^{\lambda x_{i}-1}\right)}\right)^{\alpha}\right)}, \\
& U_{\lambda}=\sum_{i=1}^{n} x_{i}+\frac{\beta}{\lambda^{2}} \sum_{i=1}^{n}\left(e^{\lambda x_{i}}-1\right) \\
& -\frac{\beta}{\lambda} \sum_{i=1}^{n} x_{i} e^{\lambda x_{i}}+(\alpha-1) \beta \sum_{i=1}^{n} \frac{\left(\lambda x_{i} e^{\lambda x_{i}}-e^{\lambda x_{i}}+1\right) e^{-\frac{\beta}{\lambda}\left(e^{\lambda x_{i}-1}\right)}}{\lambda^{2}\left(1-e^{-\frac{\beta}{\lambda}\left(e^{\lambda x_{i}-1}\right)}\right)} \\
& -2 \sum_{i=1}^{n} \frac{\alpha \beta \bar{\theta}\left(\lambda x_{i} e^{\lambda x_{i}}-e^{\lambda x_{i}}+1\right) e^{-\frac{\beta}{\lambda}\left(e^{\lambda x_{i}-1}\right)}\left(1-e^{-\frac{\beta}{\lambda}\left(e^{\lambda x_{i}-1}\right)}\right)^{\alpha-1}}{\lambda^{2}\left(\theta+\bar{\theta}\left(1-e^{-\frac{\beta}{\lambda}\left(e^{\lambda x_{i}-1}\right)}\right)^{\alpha}\right)},
\end{aligned}
$$

and

$$
U_{\theta}=\frac{n}{\theta}-2 \sum_{i=1}^{n} \frac{1-\left(1-e^{-\frac{\beta}{\lambda}\left(e^{\lambda x_{i}}-1\right)}\right)^{\alpha}}{\theta+\bar{\theta}\left(1-e^{-\frac{\beta}{\lambda}\left(e^{\lambda x_{i}}-1\right)}\right)^{\alpha}}
$$


The MLE $\widehat{\boldsymbol{\Theta}}=(\hat{\alpha}, \hat{\beta}, \hat{\lambda}, \hat{\theta})^{T}$ of $\boldsymbol{\Theta}=(\alpha, \beta, \lambda, \theta)^{T}$ is obtained by solving the nonlinear likelihood equation $U_{\alpha}=0, U_{\beta}=0, U_{\lambda}=0$ and $U_{\theta}=0$. Since these equations cannot be solved analytically, then we use the statistical software to solve them numerically via iterative methods such as a Newton-Raphson technique.

The normal approximation for the MLE of $\boldsymbol{\Theta}$ is used for constructing approximate confidence intervals, confidence regions and testing hypotheses of the parameters $\alpha, \beta, \lambda$ and $\theta$. Under conditions that are fulfilled for parameters in the interior of the parameter space but not on the boundary, the asymptotic distribution of $\sqrt{n}(\widehat{\boldsymbol{\Theta}}-\boldsymbol{\Theta})$ is $N_{4}\left(0, I^{-1}(\boldsymbol{\Theta})\right)$, where $I(\boldsymbol{\Theta})$ is the expected information matrix. This asymptotic behavior is valid if $I(\boldsymbol{\Theta})$ is replaced by $J(\widehat{\boldsymbol{\Theta}})$, where $J(\Theta)$ is the observed information matrix, given by

$$
J(\Theta)=-\left(\begin{array}{cccc}
U_{\alpha \alpha} & U_{\alpha \beta} & U_{\alpha \lambda} & U_{\alpha \theta} \\
\cdot & U_{\beta \beta} & U_{\beta \lambda} & U_{\beta \theta} \\
\cdot & \cdot & U_{\lambda \lambda} & U_{\lambda \theta} \\
\cdot & . & \cdot & U_{\theta \theta}
\end{array}\right),
$$

whose elements are given in the Appendix.

Therefore, an 100(1- $\omega) \%$ asymptotic confidence intervals for the parameters $\alpha, \beta, \lambda$ and $\theta$ are given by

$$
\hat{\alpha} \pm Z_{\frac{\omega}{2}} \sqrt{\operatorname{var}(\hat{\alpha})}, \hat{\beta} \pm Z_{\frac{\omega}{2}} \sqrt{\operatorname{var}(\hat{\beta})}, \hat{\lambda} \pm Z_{\frac{\omega}{2}} \sqrt{\operatorname{var}(\hat{\lambda})} \text { and } \hat{\theta} \pm Z_{\frac{\omega}{2}} \sqrt{\operatorname{var}(\hat{\theta})}
$$

respectively, where $\operatorname{var}(\cdot)$ is the diagonal element of $J^{-1}(\widehat{\Theta})$, corresponding to each

parameter and $Z_{\frac{\omega}{2}}$ is the quantile $(1-\omega / 2)$ of the standard normal distribution.

\section{Application}

In this section, we give an application of the MOEGG distribution to one real data set to demonstrate its superiority. We consider the data set given by Birnbaum and Saunders (1969) on the fatigue time of 6061-T6 aluminum coupons cut parallel to the direction of rolling and oscillated at 18 cycles per second (cps). The data set is: 70, 90, 96, 97, 99, 100, 103, 104, 104, $105,107,108,108,108,109,109,112,112,113,114,114,114,116,119,120,120,120,121$, $121,123,124,124,124,124,124,128,128,129,129,130,130,130,131,131,131,131,131$, 132 , 132, 132, 133, 134, 134, 134, 134, 134, 136, 136, 137, 138, 138, 138, 139, 139, 141, 141, $142,142,142,142,142,142,144,144,145,146,148,148,149,151,151,152,155,156,157$, 157, 157, 157, 158, 159, 162, 163, 163, 164, 166, 166, 168, 170, 174, 196, 212.

We compare the MOEGG model with the Gompertz, GG, beta Gompertz (BG), McDonald Gompertz (McG), Kumaraswamy Gompertz (KwG), Marshall-Olkin extend Gompertz (MOEG) and generalized exponential (GE) models. The pdf of the GG (El-Gohary et al., 2013) distribution is 


$$
f(x)=\alpha \beta e^{\lambda x} e^{-\frac{\beta}{\lambda}\left(e^{\lambda x}-1\right)}\left(1-e^{-\frac{\beta}{\lambda}\left(e^{\lambda x}-1\right)}\right)^{\alpha-1}, \quad x, \alpha, \beta, \lambda>0 .
$$

For $\alpha=1$ the GG distribution becomes the Gompertz distribution. The pdf of the BG distribution (Jafari et al., 2014) is

$$
f(x)=\frac{\beta e^{\lambda x} e^{-\frac{\gamma \beta}{\lambda}\left(e^{\lambda x}-1\right)}}{B(\theta, \gamma)}\left(1-e^{-\frac{\beta}{\lambda}\left(e^{\lambda x}-1\right)}\right)^{\theta-1}, \quad x, \beta, \lambda, \theta, \gamma>0 .
$$

The pdf of the McG distribution (Roozegar et al., 2015) is

$$
f(x)=\frac{\delta \beta e^{\lambda x} e^{-\frac{\beta}{\lambda}\left(e^{\lambda x}-1\right)}}{B(\theta / \delta, \gamma)}\left(1-e^{-\frac{\beta}{\lambda}\left(e^{\lambda x}-1\right)}\right)^{\theta-1}\left(1-\left(1-e^{-\frac{\beta}{\lambda}\left(e^{\lambda x}-1\right)}\right)^{\delta}\right)^{\gamma-1},
$$

for $x, \beta, \lambda, \theta, \gamma, \delta>0$. The pdf of the KwG distribution (da Silva et al., 2015) is

$$
f(x)=\theta \gamma \beta e^{\lambda x} e^{-\frac{\beta}{\lambda}\left(e^{\lambda x}-1\right)}\left(1-e^{-\frac{\beta}{\lambda}\left(e^{\lambda x}-1\right)}\right)^{\theta-1}\left(1-\left(1-e^{-\frac{\beta}{\lambda}\left(e^{\lambda x}-1\right)}\right)^{\theta}\right)^{\gamma-1},
$$

for $x, \beta, \lambda, \theta, \gamma>0$. The pdf of the MOEG distribution is

$$
f(x)=\frac{\beta \theta e^{\lambda x} e^{-\frac{\beta}{\lambda}\left(e^{\lambda x}-1\right)}}{\left[\theta+\bar{\theta}\left(1-e^{-\frac{\beta}{\lambda}\left(e^{\lambda x}-1\right)}\right)\right]^{2}}, \quad x, \lambda, \theta>0 .
$$

The model selection is carried out using the maximized log-likelihood $(-\ln (\mathrm{L}))$, Akaike Information Criterion (AIC), Consistent Akaike Information Criteria (CAIC), Bayesian Information Criteria (BIC), Kolmogorov-Smirnov (K-S) statistic with its respective $p$-value, Cramér-von Mises (CM) statistic and Anderson-Darling (AD) statistic. The better distribution to fit the data corresponds to smaller values of these statistics and the large $p$-value for the K-S test. The MLEs and the corresponding standard errors in parentheses are listed in Table 5. The values of the - $\ln (\mathrm{L}), \mathrm{AIC}, \mathrm{BIC}, \mathrm{CAIC}$ and K-S with its respective $p$-value are listed in Table 6 whereas the values of the $\mathrm{CM}$ and $\mathrm{AD}$ are given in Table 7. So, we conclude that the MOEGG distribution gives an excellent fit for the data set. In addition, the plots of the densities together with the data histogram (Figure 3), cdfs with empirical distribution function (Figure 4) and the probability plots (Figure 5) confirm that the MOEGG model yields a better fit.

The asymptotic variance-covariance matrix of the MLEs of the MOEGG model parameters, which is the inverse of the observed information matrix $J^{-1}(\widehat{\boldsymbol{\Theta}})$, is

$$
\left(\begin{array}{cccl}
7.6787 \times 10^{-13} & -2.7685 \times 10^{-9} & 1.1529 \times 10^{-9} & 2.1213 \times 10^{-12} \\
-2.7685 \times 10^{-9} & 1.0026 \times 10^{-5} & -4.1536 \times 10^{-6} & -7.6789 \times 10^{-9} \\
1.1529 \times 10^{-9} & -4.1536 \times 10^{-6} \cdot & 1.8129 \times 10^{-6} & 3.1946 \times 10^{-9} \\
2.1213 \times 10^{-12} & -7.6789 \times 10^{-9} & 3.1946 \times 10^{-9} & 5.8834 \times 10^{-12}
\end{array}\right) \text {. }
$$


Therefore, the 95\% asymptotic confidence intervals for $\alpha, \beta, \lambda$ and $\theta$ are $42.1460 \pm$ $1.7142 \times 10^{-6}, 0.0281 \pm 0.0062,0.0069 \pm 4.7541 \times 10^{-6}$ and $10.9770 \pm 0.0026$ respectively.

Table 5: MLEs of the model parameters and the corresponding standard errors given in parentheses.

\begin{tabular}{lllllll}
\hline Model & $\alpha$ & $\beta$ & $\lambda$ & $\theta$ & $\gamma$ & $\delta$ \\
\hline MOEGG & 42.1460 & 0.0281 & 0.0069 & 10.9770 & - & - \\
MOEG & $\left(8.7457 \times 10^{-7}\right)$ & $(0.0032)$ & $(0.0013)$ & $\left(2.4256 \times 10^{-6}\right)$ & - & - \\
& - & 0.0071 & 0.0161 & 26.342 & - & - \\
KwG & - & $(0.0013)$ & $(0.0018)$ & $\left(5.2376 \times 10^{-7}\right)$ & - & - \\
& - & 0.0066 & 0.0125 & 9.9103 & 1.7043 & - \\
BG & - & $(0.0010)$ & $(0.0041)$ & $(0.1768)$ & $(1.2965)$ & - \\
& - & 0.0081 & 0.0196 & 15.4744 & 0.3253 & - \\
McG & - & $(0.0016)$ & $(0.0041)$ & $(0.00096)$ & $(0.1693)$ & - \\
& - & 0.0045 & 0.0148 & 6.3659 & 1.7887 & 9.4524 \\
GG & - & $(0.00072)$ & $(0.00315)$ & $(0.3446)$ & $(1.0539)$ & $(0.0547)$ \\
& 0.7901 & 0.0014 & 0.0183 & - & - & - \\
GE & $(0.0982)$ & $(0.000456)$ & $(0.0027)$ & - & - & - \\
& 174.6500 & 0.0421 & - & - & - & - \\
Gompertz & $\left(3.9676 \times 10^{-8}\right)$ & $(0.00086)$ & - & - & - & - \\
& - & 0.0010 & 0.0229 & - & - & - \\
\hline
\end{tabular}


L. Benkhelifa 259

Table 6: The statistics: $-\ln (\mathrm{L}), \mathrm{AIC}, \mathrm{CAIC}, \mathrm{BIC}, \mathrm{K}-\mathrm{S}$ and p-values.

\begin{tabular}{lllllll}
\hline Model & $-\ln (\mathrm{L})$ & AIC & CAIC & BIC & K-S & p-value \\
\hline MOEGG & 456.1970 & 920.3940 & 920.8107 & 930.8545 & 0.0599 & 0.8614 \\
KwG & 456.7241 & 921.4482 & 921.8649 & 931.9087 & 0.06738 & 0.7488 \\
BG & 457.5082 & 923.0164 & 923.4331 & 933.4769 & 0.0798 & 0.5409 \\
McG & 458.1217 & 926.2435 & 926.8751 & 939.3191 & 0.0765 & 0.5957 \\
MOEG & 465.4312 & 936.8624 & 937.1098 & 944.7078 & 0.1143 & 0.1431 \\
GG & 516.6602 & 1039.32 & 1039.568 & 994.2344 & 0.3730 & $1.25 \times$ \\
GE & 463.4165 & 930.833 & 930.9554 & 936.0632 & 0.10291 & 0.2351 \\
Gompertz & 492.5021 & 989.0041 & 989.1266 & 1047.166 & 0.29477 & $4.78 \times 10^{-8}$ \\
\hline
\end{tabular}

Table 7: The statistics CM and AD.

\begin{tabular}{|l|l|l|}
\hline Model & CM & AD \\
\hline MOEGG & 0.0473 & 0.3513 \\
\hline KwG & 0.0695 & 0.4701 \\
\hline BG & 0.1003 & 0.6288 \\
\hline McG & 0.1012 & 0.7219 \\
\hline MOEG & 0.2639 & 2.0088 \\
\hline GG & 4.1928 & 20.2110 \\
\hline GE & 0.9843 & 5.8964 \\
\hline Gompertz & 2.6406 & 13.3170 \\
\hline
\end{tabular}




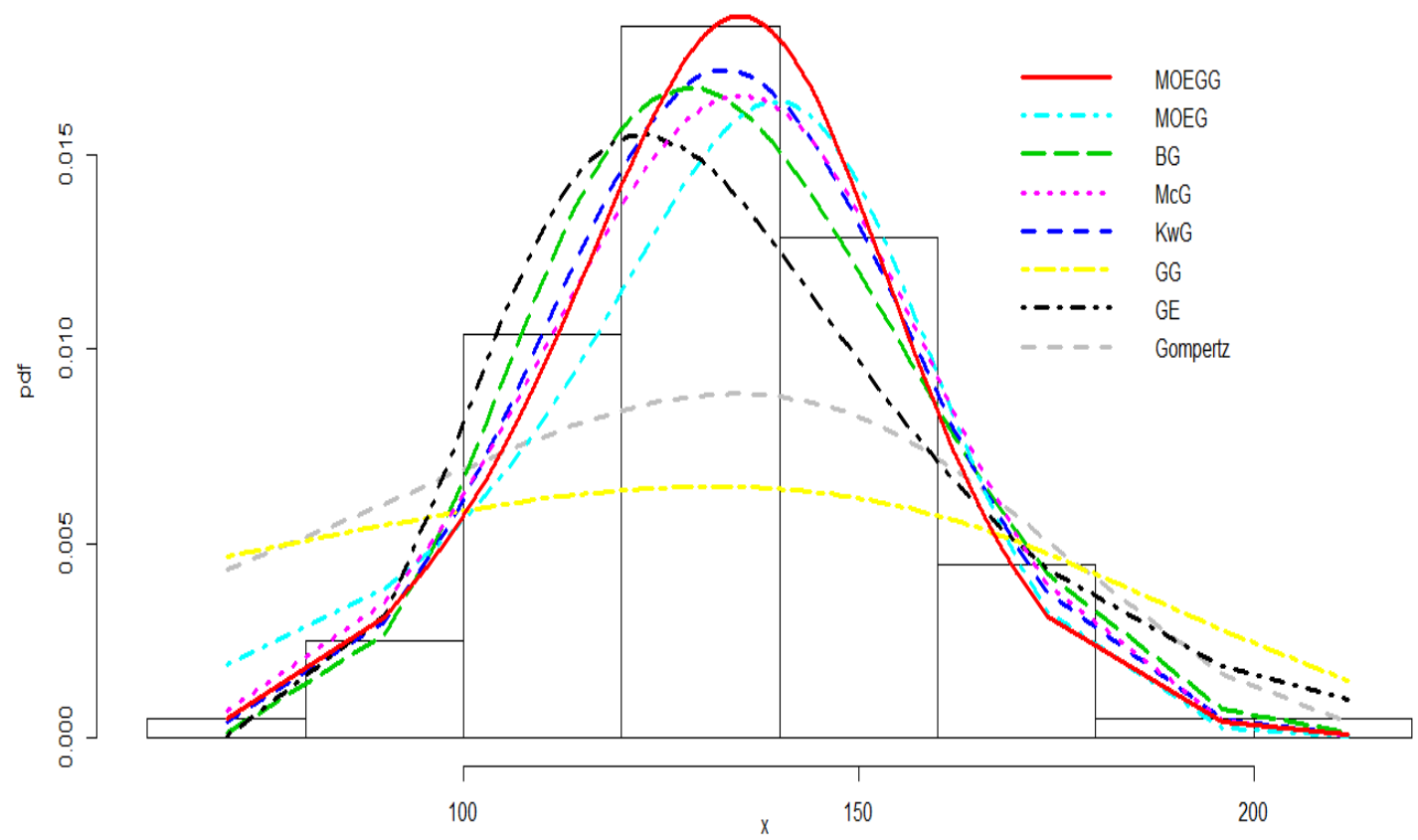

Figure 3. Histogram and estimated densities.
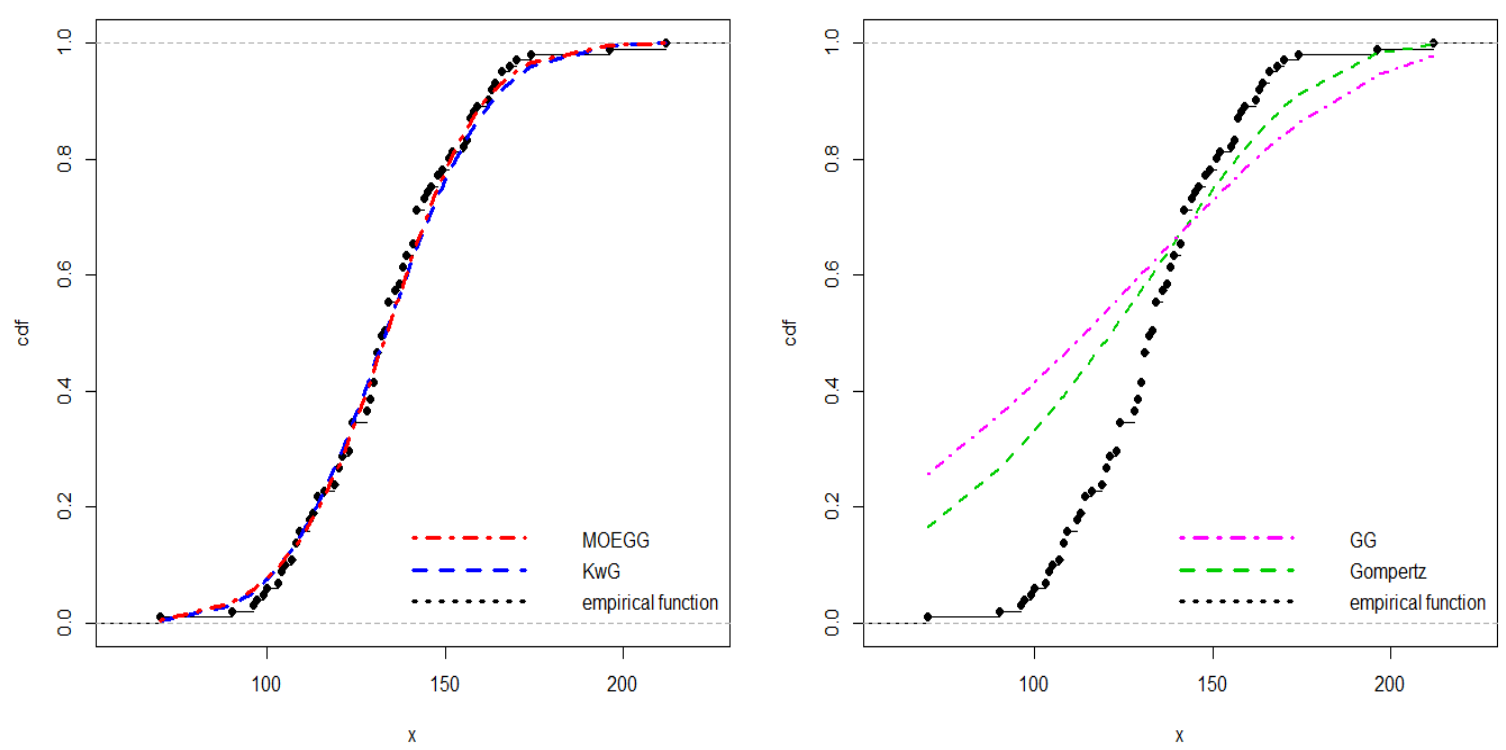

Figure 4. The estimated cdfs and the empirical function. 

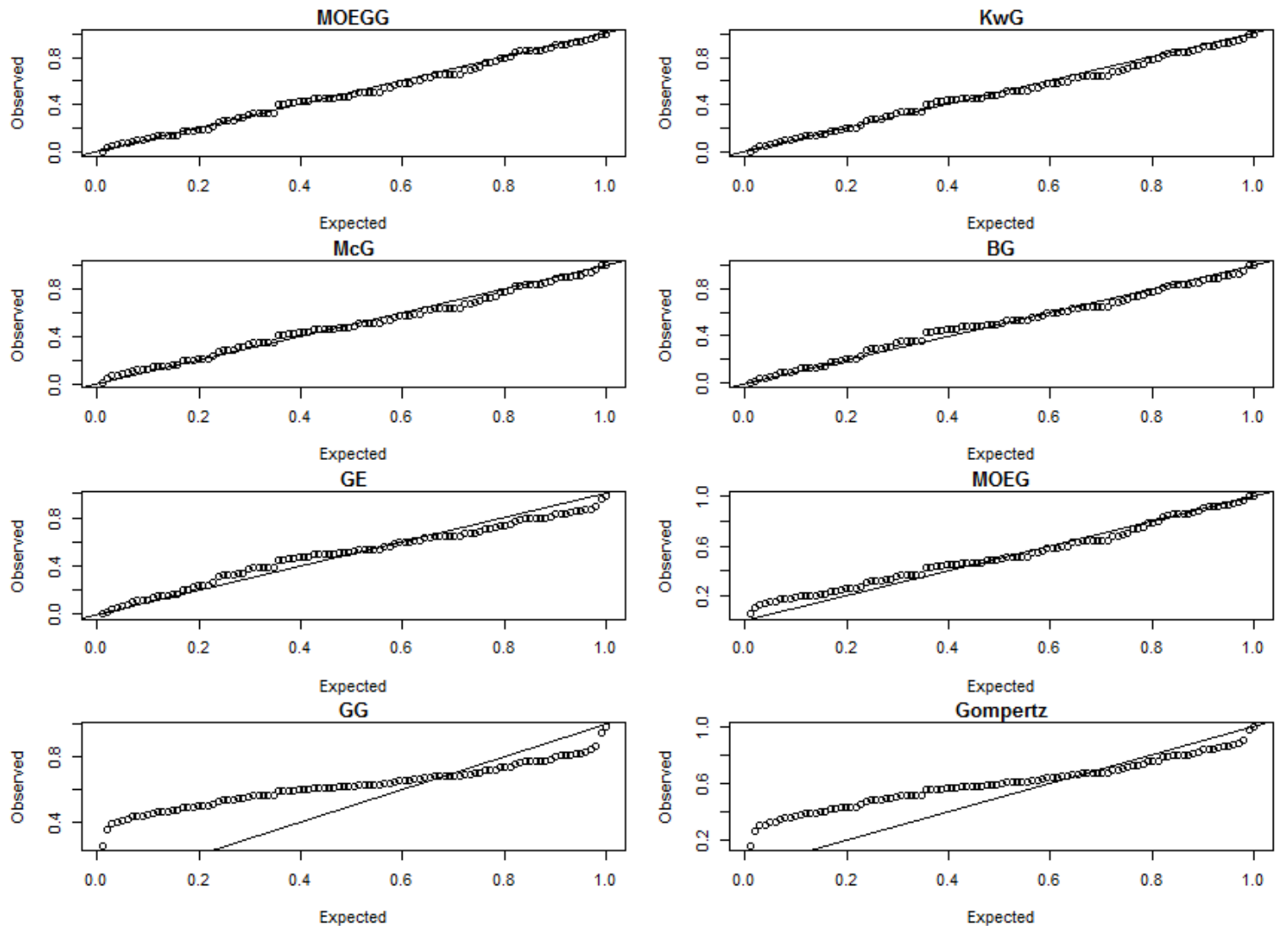

Figure 5. The probability plots of the fitted models.

\section{Conclusion}

In this paper, we have proposed a new four-parameter continuous distribution called the Marshall-Olkin extended generalized Gompertz distribution. This distribution is generated by using the Marshall and Olkin method. It includes as special sub-models the Gompertz, exponential, generalized Gompertz, generalized exponential, Marshall-Olkin extended Gompertz, Marshall-Olkin extended generalized exponential and Marshall-Olkin extended exponential distributions. We have provided some mathematical properties of the proposed model. The estimation of the unknown parameters of this model is approached by the method of maximum likelihood and the observed information matrix is derived. We have presented an application to real data to illustrate the application of the proposed model. The results of this application suggest that the new model provides consistently better fit. We hope that the new proposed model may attract wider applications in several fields. 


\section{Appendix: Observed information matrix}

Let $z_{i}=e^{-\frac{\beta}{\lambda}\left(e^{\lambda x_{i}}-1\right)}$. The elements of the observed information matrix $J(\boldsymbol{\Theta})$ are

$$
\begin{aligned}
& U_{\alpha \alpha}=-\frac{n}{\alpha^{2}}-2 \bar{\theta} \sum_{i=1}^{n} \frac{\left(1-z_{i}\right)^{\alpha} \ln \left(1-z_{i}\right)}{\theta+\bar{\theta}\left(1-z_{i}\right)^{\alpha}}+2 \bar{\theta}^{2} \sum_{i=1}^{n}\left(\frac{\left(1-z_{i}\right)^{\alpha} \ln \left(1-z_{i}\right)}{\theta+\bar{\theta}\left(1-z_{i}\right)^{\alpha}}\right)^{2} \text {, } \\
& U_{\alpha \beta}=\frac{1}{\lambda} \sum_{i=1}^{n} \frac{\left(e^{\lambda x_{i}}-1\right) z_{i}}{1-z_{i}}-\frac{2 \alpha \bar{\theta}}{\lambda} \sum_{i=1}^{n} \frac{\left(e^{\lambda x_{i}}-1\right) z_{i}\left(1-z_{i}\right)^{\alpha-1} \ln \left(1-z_{i}\right)}{\theta+\bar{\theta}\left(1-z_{i}\right)^{\alpha}} \\
& -\frac{2 \bar{\theta}}{\lambda} \sum_{i=1}^{n} \frac{\left(e^{\lambda x_{i}}-1\right) z_{i}}{\left(1-z_{i}\right)\left[\theta+\bar{\theta}\left(1-z_{i}\right)^{\alpha}\right]^{2}} \\
& +\frac{2 \alpha \bar{\theta}^{2}}{\lambda} \sum_{i=1}^{n} \frac{\left(e^{\lambda x_{i}}-1\right) z_{i}\left(1-z_{i}\right)^{2 \alpha-1} \ln \left(1-z_{i}\right)}{\left[\theta+\bar{\theta}\left(1-z_{i}\right)^{\alpha}\right]^{2}} \\
& U_{\alpha \lambda}=\frac{\beta}{\lambda} \sum_{i=1}^{n} \frac{\left(\lambda x_{i} e^{\lambda x_{i}}-e^{\lambda x_{i}}+1\right) z_{i}}{1-z_{i}} \\
& -\frac{2 \beta \bar{\theta}}{\lambda} \sum_{i=1}^{n} \frac{\left[1+\alpha \ln \left(1-z_{i}\right)\right]\left(\lambda x_{i} e^{\lambda x_{i}}-e^{\lambda x_{i}}+1\right) z_{i}\left(1-z_{i}\right)^{\alpha-1}}{\theta+\bar{\theta}\left(1-z_{i}\right)^{\alpha}}, \\
& U_{\alpha \theta}=2 \sum_{i=1}^{n} \frac{\left(1-z_{i}\right)^{\alpha} \ln \left(1-z_{i}\right)}{\left[\theta+\bar{\theta}\left(1-z_{i}\right)^{\alpha}\right]^{2}}, \\
& U_{\beta \beta}=-\frac{n}{\beta^{2}}-\frac{(\alpha-1)}{\lambda^{2}} \sum_{i=1}^{n} \frac{\left(e^{\lambda x_{i}}-1\right)^{2} z_{i}}{1-z_{i}}-\frac{(\alpha-1)}{\lambda^{2}} \sum_{i=1}^{n} \frac{z_{i}^{2}\left(e^{\lambda x_{i}}-1\right)^{2}}{\left(1-z_{i}\right)^{2}} \\
& +\frac{2 \bar{\theta} \alpha}{\lambda^{2}} \sum_{i=1}^{n} \frac{\left(e^{\lambda x_{i}}-1\right)^{2} z_{i}\left(1-z_{i}\right)^{\alpha-1}}{\theta+\bar{\theta}\left(1-z_{i}\right)^{\alpha}} \\
& -\frac{2 \bar{\theta} \alpha(\alpha-1)}{\lambda^{2}} \sum_{i=1}^{n} \frac{\left(e^{\lambda x_{i}}-1\right)^{2} z_{i}^{2}\left(1-z_{i}\right)^{\alpha-2}}{\theta+\bar{\theta}\left(1-z_{i}\right)^{\alpha}} \\
& +\frac{2 \bar{\theta}^{2} \alpha^{2}}{\lambda^{2}} \sum_{i=1}^{n}\left(\frac{\left(e^{\lambda x_{i}}-1\right) z_{i}\left(1-z_{i}\right)^{\alpha-1}}{\theta+\bar{\theta}\left(1-z_{i}\right)^{\alpha}}\right)^{2} \text {, }
\end{aligned}
$$




$$
\begin{aligned}
U_{\beta \lambda}=\frac{1}{\lambda^{2}} \sum_{i=1}^{n}( & \left.e^{\lambda x_{i}}-1\right)-\frac{1}{\lambda} \sum_{i=1}^{n} x_{i} e^{\lambda x_{i}}+\frac{(\alpha-1)}{\lambda^{2}} \sum_{i=1}^{n} \frac{\left(\lambda-\beta e^{\lambda x_{i}}+\beta\right) z_{i} x_{i} e^{\lambda x_{i}}}{1-z_{i}} \\
& +\frac{\beta(\alpha-1)}{\lambda^{3}} \sum_{i=1}^{n} \frac{\left(e^{\lambda x_{i}}-1\right)^{2} z_{i}}{1-z_{i}}-\frac{(\alpha-1)}{\lambda} \sum_{i=1}^{n} \frac{\left(e^{\lambda x_{i}}-1\right) z_{i}}{1-z_{i}} \\
& -\frac{\beta(\alpha-1)}{\lambda^{3}} \sum_{i=1}^{n} \frac{\left(\lambda x_{i} e^{\lambda x_{i}}-e^{\lambda x_{i}}+1\right)\left(e^{\lambda x_{i}}-1\right) z_{i}^{2}}{\left(1-z_{i}\right)^{2}} \\
& -\frac{2 \bar{\theta} \alpha}{\lambda} \sum_{i=1}^{n} \frac{x_{i} e^{\lambda x_{i}} z_{i}\left(1-z_{i}\right)^{\alpha-1}}{\theta+\bar{\theta}\left(1-z_{i}\right)^{\alpha}} \\
& -\frac{2 \bar{\theta} \alpha \beta}{\lambda^{3}} \sum_{i=1}^{n} \frac{\left(\lambda x_{i} e^{\lambda x_{i}}-e^{\lambda x_{i}}+1\right)\left(e^{\lambda x_{i}}-1\right) z_{i}\left(1-z_{i}\right)^{\alpha-1}}{\theta+\bar{\theta}\left(1-z_{i}\right)^{\alpha}} \\
& -\frac{2 \bar{\theta} \alpha \beta(\alpha-1)}{\lambda^{3}} \frac{n}{n} \frac{\left(\lambda x_{i} e^{\lambda x_{i}}-e^{\lambda x_{i}}+1\right)\left(e^{\lambda x_{i}}-1\right) z_{i}^{2}\left(1-z_{i}\right)^{\alpha-2}}{\theta+\bar{\theta}\left(1-z_{i}\right)^{\alpha}} \\
& +\frac{2 \bar{\theta} \alpha}{\lambda^{2}} \sum_{i=1}^{n} \frac{\left(e^{\lambda x_{i}}-1\right) z_{i}\left(1-z_{i}\right)^{\alpha-1}}{\theta+\bar{\theta}\left(1-z_{i}\right)^{\alpha}} \\
& +\frac{2 \bar{\theta} \alpha \beta}{\lambda^{3}} \sum_{i=1}^{n} \frac{\left(\lambda x_{i} e^{\lambda x_{i}}-e^{\lambda x_{i}}+1\right)\left(e^{\lambda x_{i}}-1\right) z_{i}^{2}\left(1-z_{i}\right)^{2 \alpha-2}}{\left[\theta+\bar{\theta}\left(1-z_{i}\right)^{\alpha}\right]^{2}}
\end{aligned}
$$




$$
\begin{aligned}
& U_{\lambda \lambda}=-\frac{2 \beta}{\lambda^{3}} \sum_{i=1}^{n}\left(e^{\lambda x_{i}}-1\right)+\frac{2 \beta}{\lambda^{2}} \sum_{i=1}^{n} x_{i} e^{\lambda x_{i}}-\frac{\beta}{\lambda} \sum_{i=1}^{n} x_{i}^{2} e^{\lambda x_{i}}+\frac{\beta(\alpha-1)}{\lambda} \sum_{i=1}^{n} \frac{z_{i} x_{i}^{2} e^{\lambda x_{i}}}{1-z_{i}} \\
& -\frac{\beta^{2}(\alpha-1)}{\lambda^{4}} \sum_{i=1}^{n} \frac{\left(\lambda x_{i} e^{\lambda x_{i}}-e^{\lambda x_{i}}+1\right)^{2} z_{i}}{1-z_{i}} \\
& -\frac{2 \beta(\alpha-1)}{\lambda^{3}} \sum_{i=1}^{n} \frac{\left(\lambda x_{i} e^{\lambda x_{i}}-e^{\lambda x_{i}}+1\right) z_{i}}{1-z_{i}} \\
& -\frac{\beta^{2}(\alpha-1)}{\lambda^{4}} \sum_{i=1}^{n}\left(\frac{\left(\lambda x_{i} e^{\lambda x_{i}}-e^{\lambda x_{i}}+1\right) z_{i}}{1-z_{i}}\right)^{2} \\
& -2 \alpha \beta \bar{\theta} \sum_{i=1}^{n} \frac{x_{i}^{2} e^{\lambda x_{i}} z_{i}\left(1-z_{i}\right)^{\alpha-1}}{\theta+\bar{\theta}\left(1-z_{i}\right)^{\alpha}} \\
& +\frac{2 \alpha \beta^{2} \bar{\theta}}{\lambda^{3}} \sum_{i=1}^{n} \frac{\left(\lambda x_{i} e^{\lambda x_{i}}-e^{\lambda x_{i}}+1\right)^{2} z_{i}\left(1-z_{i}\right)^{\alpha-1}}{\theta+\bar{\theta}\left(1-z_{i}\right)^{\alpha}} \\
& -\frac{2 \beta^{2} \bar{\theta} \alpha(\alpha-1)}{\lambda^{3}} \sum_{i=1}^{n} \frac{\left(\lambda x_{i} e^{\lambda x_{i}}-e^{\lambda x_{i}}+1\right)^{2} z_{i}^{2}\left(1-z_{i}\right)^{\alpha-2}}{\theta+\bar{\theta}\left(1-z_{i}\right)^{\alpha}} \\
& +\frac{4 \beta \alpha \bar{\theta}}{\lambda^{3}} \sum_{i=1}^{n} \frac{\left(\lambda x_{i} e^{\lambda x_{i}}-e^{\lambda x_{i}}+1\right) z_{i}\left(1-z_{i}\right)^{\alpha-1}}{\theta+\bar{\theta}\left(1-z_{i}\right)^{\alpha}} \\
& +\frac{2(\alpha \beta \bar{\theta})^{2}}{\lambda^{4}} \sum_{i=1}^{n}\left(\frac{\left(\lambda x_{i} e^{\lambda x_{i}}-e^{\lambda x_{i}}+1\right) z_{i}\left(1-z_{i}\right)^{\alpha-1}}{\theta+\bar{\theta}\left(1-z_{i}\right)^{\alpha}}\right)^{2}, \\
& U_{\beta \theta}=\frac{2 \alpha}{\lambda} \sum_{i=1}^{n} \frac{\left(e^{\lambda x_{i}}-1\right) z_{i}\left(1-z_{i}\right)^{\alpha-1}}{\theta+\bar{\theta}\left(1-z_{i}\right)^{\alpha}} \\
& +2 \alpha \bar{\theta} \sum_{i=1}^{n} \frac{\left[1-\left(1-z_{i}\right)^{\alpha}\right]\left(e^{\lambda x_{i}}-1\right) z_{i}\left(1-z_{i}\right)^{\alpha-1}}{\left[\theta+\bar{\theta}\left(1-z_{i}\right)^{\alpha}\right]^{2}},
\end{aligned}
$$

and

$$
U_{\theta \theta}=-\frac{n}{\theta^{2}}+2 \sum_{i=1}^{n}\left(\frac{1-\left(1-z_{i}\right)^{\alpha}}{\theta+\bar{\theta}\left(1-z_{i}\right)^{\alpha}}\right)^{2} \text {. }
$$




\section{Acknowledgments}

The author would like to express his sincere thanks to the editor and the referee for their useful comments and suggestions.

\section{References}

[1] Alexander C., Cordeiro G. M. and Ortega E. M. M., Sarabia J. M. (2012). Generalized beta-generated distributions. Computational Statistics and Data Analysis 56, 18801897.

[2] Al-Saiari A. Y., Baharith L. A. and Mousa S. A. (2014). Marshall-Olkin extended Burr type XII distribution. International Journal of Statistics and Probability 3, 78-84.

[3] Benkhelifa L. (2017). The Marshall-Olkin extended generalized Lindley distribution: properties and applications. Communications in Statistics-Simulation and Computation. http://dx.doi.org/10.1080/03610918.2016.1277747.

[4] Birnbaum Z. W. and Saunders S. C. (1969). Estimation for a family of life distributions with applications to fatigue. Journal of Applied Probability 6, 328-347.

[5] Cordeiro G. M., Lemonte A. J. and Ortega E. M. M. (2014). The Marshall-Olkin family of distributions: mathematical properties and new models. Journal of Statistical Theory and Practice 8, 343-366.

[6] El-Gohary A., Alshamrani A. and Al-Otaibi A. (2013). The generalized Gompertz distribution. Applied Mathematical Modelling 37, 13-24.

[7] da Silva R. C., Sanchez J. J. D., Lima F. P. and Cordeiro G. M. (2015). The Kumaraswamy Gompertz distribution. Journal of Data Science 13, 241-260.

[8] Eugene N., Lee C., Famoye F. (2002). Beta-normal distribution and its applications. Communications in Statistics-Theory and Methods 31, 497-512.

[9] García V. J., Gómez-Déniz E. and Vázquez-Polo F. J. (2010). A new skew generalization of the normal distribution: Properties and applications. Computational Statistics and Data Analysis 54, 2021-2034.

[10] Ghitany M. E., AL-Hussaini E. K. and AL Jarallah R. A. (2005). Marshall-Olkin extended Weibull distribution and its application to censored data. Journal of Applied Statistics 32, 1025-1034. 
[11] Gompertz B. (1825). On the nature of the function expressive of the law of human mortality and on the new mode of determining the value of life contingencies. Philosophical Transactions of the Royal Statistical Society 115, 513-580.

[12] Jafari A. A., Tahmasebi S. and Alizadeh M. (2014). The beta-Gompertz distribution. Revista Colombiana de Estadística 37, 141-158.

[13] Johnson N. L., Kotz S. and Balakrishnan N. (1995). Continuous univariate distributions. John Wiley \& Sons, New York, volume 2, second edition.

[14] Kumar D. (2016). Ratio and inverse moments of Marshall-Olkin extended Burr type XII distribution based on lower generalized order statistics. Journal of Data Science $14,53-66$.

[15] Lehmann E. E. (1953). The power of rank tests. Annals of Mathematical Statistics 24, 23-43.

[16] Marshall A.W. and Olkin I. (1997). A new method for adding a parameter to a family of distributions with application to the exponential and Weibull families. Biometrika 84, 641-652.

[17] Milgram M. (1985). The generalized integro-exponential function. Mathematics of Computation 44, 443-458.

[18] Okasha H. M. and Kayid M. (2016). A new family of Marshall-Olkin extended generalized linear exponential distribution. Journal of Computational and Applied Mathematics 296, 576-592.

[19] Roozegar R., Tahmasebi S. and Jafari A. A. (2015). The McDonald Gompertz distribution: properties and applications. Communications in Statistics-Simulation and Computation. http://dx.doi.org/10.1080/03610918.2015.1088024.

[20] RistícM.M.andKundu D.(2015).Marshall-Olkingeneralizedexponential distribution. Metron 73, 317-333.

[21] Shaked M., Shanthikumar J. G. (2007). Stochastic orders. Springer, New York.

Lazhar Benkhelifa

Laboratory of Applied Mathematics

Mohamed Khider University,

Biskra 07000, Algeria

Department of Mathematics and Informatics

Larbi Ben M'Hidi University

Oum El Bouaghi, 04000, Algeria

E-mail: 1.benkhelifa@yahoo.fr 